\title{
First axion results from the XENON100 experiment
}

E. Aprile, ${ }^{1}$ F. Agostini, ${ }^{2,}$ M. Alfonsi, ${ }^{3}$ K. Arisaka,${ }^{4}$ F. Arneodo, ${ }^{5,}$ M. Auger, ${ }^{6}$ C. Balan, ${ }^{7}$ P. Barrow,${ }^{6}$ L. Baudis, ${ }^{6}$ B. Bauermeister, ${ }^{8}$ A. Behrens, ${ }^{6}$ P. Beltrame, ${ }^{9,}$ K. Bokeloh, ${ }^{10}$ A. Brown, ${ }^{11}$ E. Brown, ${ }^{10}$ S. Bruenner, ${ }^{12}$ G. Bruno, ${ }^{5}$ R. Budnik, ${ }^{9}$ J. M. R. Cardoso, ${ }^{7}$ A. P. Colijn, ${ }^{3}$ H. Contreras, ${ }^{1}$ J. P. Cussonneau, ${ }^{13}$ M. P. Decowski, ${ }^{3}$ E. Duchovni, ${ }^{9}$ S. Fattori, ${ }^{8}$ A. D. Ferella, ${ }^{5}$ W. Fulgione, ${ }^{14}$ F. Gao,${ }^{15}$ M. Garbini, ${ }^{2}$ C. Geis, ${ }^{8}$ L. W. Goetzke, ${ }^{1}$ C. Grignon, ${ }^{8}$ E. Gross,${ }^{9}$ W. Hampel, ${ }^{12}$ R. Itay, ${ }^{9}$ F. Kaether, ${ }^{12}$ G. Kessler, ${ }^{6}$ A. Kish, ${ }^{9}$ H. Landsman, ${ }^{9}$ R. F. Lang, ${ }^{11}$ M. Le Calloch, ${ }^{13}$ D. Lellouch, ${ }^{9}$ C. Levy, ${ }^{10}$ S. Lindemann, ${ }^{12}$ M. Lindner, ${ }^{12}$ J. A. M. Lopes, ${ }^{7,}$ K. Lung, ${ }^{4}$ A. Lyashenko, ${ }^{4}$ S. MacMullin, ${ }^{11}$ T. Marrodán Undagoitia, ${ }^{12}$ J. Masbou, ${ }^{13}$ F. V. Massoli, ${ }^{2}$ D. Mayani Paras, ${ }^{6}$ A. J. Melgarejo Fernandez, ${ }^{1}$ Y. Meng, ${ }^{4}$ M. Messina, ${ }^{1}$ B. Miguez, ${ }^{14}$ A. Molinario, ${ }^{14}$ M. Murra, ${ }_{10}$ J. Naganoma, ${ }^{16} \mathrm{~K}$. Ni, ${ }^{15}$ U. Oberlack, ${ }^{8}$ S. E. A. Orrigo, ${ }^{7, \|}$ E. Pantic, ${ }^{4}$ R. Persiani, ${ }^{2}$ F. Piastra, ${ }^{6}$ J. Pienaar, ${ }^{11}$ G. Plante, ${ }^{1}$ N. Priel, ${ }^{9,9}$ S. Reichard,${ }^{11}$ C. Reuter, ${ }^{11}$ A. Rizzo, ${ }^{1}$ S. Rosendahl, ${ }^{10}$ J. M. F. dos Santos, ${ }^{7}$ G. Sartorelli, ${ }^{2}$ S. Schindler, ${ }^{8}$ J. Schreiner, ${ }^{12}$ M. Schumann, ${ }^{17}$ L. Scotto Lavina, ${ }^{13}$ M. Selvi, ${ }^{2}$ P. Shagin, ${ }^{16}$ H. Simgen, ${ }^{12}$ A. Teymourian, ${ }^{4}$ D. Thers, ${ }^{13}$ A. Tiseni, ${ }^{3}$ G. Trinchero, ${ }^{14}$ O. Vitells, ${ }^{9}$ H. Wang, ${ }^{4}$ M. Weber, ${ }^{12}$ and C. Weinheimer ${ }^{10}$

(XENON100 Collaboration)

\author{
${ }^{1}$ Department of Physics, Columbia University, 538 West 120th Street, 704 Pupin Hall, MC 5255 New York, \\ New York 10027, USA \\ ${ }^{2}$ University of Bologna and INFN-Bologna, Bologna, Italy \\ ${ }^{3}$ Nikhef and the University of Amsterdam, Science Park, Amsterdam, Netherlands \\ ${ }^{4}$ Physics \& Astronomy Department, University of California, Los Angeles, 475 Portola Plaza, Los Angeles, \\ California 90095-1547, USA \\ ${ }^{5}$ INFN, Laboratori Nazionali del Gran Sasso, Assergi (AQ), Italy \\ ${ }^{6}$ Physics Institute, University of Zürich, Zürich, Switzerland \\ ${ }^{7}$ Department of Physics, University of Coimbra, Coimbra, Portugal \\ ${ }^{8}$ Institut für Physik \& Exzellenzcluster PRISMA, Johannes Gutenberg-Universität Mainz, Mainz, Germany \\ ${ }^{9}$ Department of Particle Physics and Astrophysics, Weizmann Institute of Science, Rehovot, Israel \\ ${ }^{10}$ Institut für Kernphysik, Wilhelms-Universität Münster, Münster, Germany \\ ${ }^{11}$ Department of Physics, Purdue University, 525 Northwestern Avenue, West Lafayette, \\ Indiana 47907-2036, USA \\ ${ }^{12}$ Max-Planck-Institut für Kernphysik, Heidelberg, Germany \\ ${ }^{13}$ SUBATECH, Ecole des Mines de Nantes, CNRS/In2p3, Université de Nantes, Nantes, France \\ ${ }^{14}$ INFN-Torino and Osservatorio Astrofisico di Torino, Torino, Italy \\ ${ }^{15}$ Department of Physics \& Astronomy, Shanghai Jiao Tong University, Shanghai, China \\ ${ }^{16}$ Department of Physics and Astronomy, Rice University, 6100 Main MS-61 Houston, Texas 77005-1827, USA \\ ${ }^{17}$ Albert Einstein Center for Fundamental Physics, University of Bern, Bern, Switzerland
}

(Received 8 April 2014; published 9 September 2014)

We present the first results of searches for axions and axionlike particles with the XENON100 experiment. The axion-electron coupling constant, $g_{\mathrm{Ae}}$, has been probed by exploiting the axioelectric effect in liquid xenon. A profile likelihood analysis of 224.6 live days $\times 34-\mathrm{kg}$ exposure has shown no evidence for a signal. By rejecting $g_{\mathrm{Ae}}$ larger than $7.7 \times 10^{-12}(90 \%$ C.L.) in the solar axion search, we set the best limit to date on this coupling. In the frame of the DFSZ and KSVZ models, we exclude QCD axions heavier than 0.3 and $80 \mathrm{eV} / \mathrm{c}^{2}$, respectively. For axionlike particles, under the assumption that they constitute the whole abundance of dark matter in our galaxy, we constrain $g_{\mathrm{Ae}}$ to be lower than $1 \times 10^{-12}$ (90\% C.L.) for masses between 5 and $10 \mathrm{keV} / \mathrm{c}^{2}$.

DOI: 10.1103/PhysRevD.90.062009

PACS numbers: 95.35.+d, 95.30.Cq, 12.60.-i, 98.80.-k

*Also at GSSI, INFN, L’Aquila, Italy.

${ }^{\dagger}$ Present address: New York University, Abu Dhabi, UAE.

*Present address: SoPA, The University of Edinburgh, Edinburgh, United Kingdom.

paolo.beltrame@ed.ac.uk

${ }^{\S}$ Also at Coimbra Engineering Institute, Coimbra, Portugal.

"Present address: IFIC, CSIC-Universidad de Valencia,

Valencia, Spain.

"nadav.priel@weizmann.ac.il

\section{INTRODUCTION}

Axions were introduced in the Peccei-Quinn solution of the strong $\mathrm{CP}$ problem as pseudo-Nambu-Goldstone bosons emerging from the breaking of a global U(1) symmetry [1-3]. Although this original model has been ruled out, "invisible" axions arising from a higher 
symmetry-breaking energy scale are still allowed, as described, for example, in the DFSZ and KSVZ models [4-7]. In addition to QCD axions, axionlike particles (ALPs) are pseudoscalars that do not necessarily solve the strong $\mathrm{CP}$ problem, but that have been introduced by many extensions of the Standard Model of particle physics. Axions, as well as ALPs, are well-motivated cold dark matter candidates [8].

Astrophysical observations are thought to be the most sensitive technique for detecting axions and ALPs [9]: the Sun would constitute an intense source of this particles (referred to as solar axions), where they can be produced via Bremsstrahlung, Compton scattering, axiorecombination, and axiode-excitation [10]. Additionally, searches can be conducted for ALPs that may have been generated via a nonthermal production mechanism in the early universe and that now constitute the dark matter in our galaxy (referred to as galactic ALPs). Axions and ALPs may give rise to observable signatures in detectors through their coupling to photons $\left(g_{A \gamma}\right)$, electrons $\left(g_{\mathrm{Ae}}\right)$, and nuclei $\left(g_{A N}\right)$. The coupling $g_{\mathrm{Ae}}$ may be tested via scattering off the electron of a target, such as liquid xenon (LXe) through the axioelectric effect [11-15]. This process is the analogue of the photoelectric process with the absorption of an axion instead of a photon.

We report on the first axion searches performed with the XENON100 experiment. The expected interaction rate is obtained by the convolution of the flux and the axioelectric cross section. The latter is given, both for QCD axions and ALPs, by

$$
\sigma_{\mathrm{Ae}}=\sigma_{p e}\left(E_{A}\right) \frac{g_{\mathrm{Ae}}^{2}}{\beta_{A}} \frac{3 E_{A}^{2}}{16 \pi \alpha_{e m} m_{e}^{2}}\left(1-\frac{\beta_{A}^{2 / 3}}{3}\right),
$$

as described in [12-16]. In Eq. (1), $\sigma_{p e}$ is the photoelectric cross section for LXe [17], $E_{A}$ is the axion energy, $\alpha_{e m}$ is the fine structure constant, $m_{e}$ is the electron mass, and $\beta_{A}$ is the axion velocity over the speed of light, $c$.

The solar axion flux has recently been recalculated in [10]. This incorporates four production mechanisms that depend on $g_{\mathrm{Ae}}$ : Bremsstrahlung, Compton scattering, atomic recombination, and atomic de-excitation. The corresponding flux is $30 \%$ larger than previous estimates due to atomic recombination and de-excitation, which previously were not taken into account. However, [10] does not include corrections for axions heavier than $1 \mathrm{keV} / \mathrm{c}^{2}$, which we therefore takes as an upper mass limit for our analysis. For solar axions, both flux and cross section depend on $g_{\mathrm{Ae}}^{2}$; thus, the interaction rate scales with the fourth power of the coupling.

For nonrelativistic ALPs in the galaxy, assuming that they constitute the whole dark matter halo density $\left(\rho_{\mathrm{DM}} \sim 0.3 \mathrm{GeV} / \mathrm{cm}^{3}[18]\right)$, the total flux is given by $\phi_{\mathrm{ALP}}=c \beta_{A} \times \rho_{\mathrm{DM}} / m_{A}$, where $m_{A}$ is the ALP mass. The interaction rate for these ALPs depends on $g_{\mathrm{Ae}}^{2}$, as the flux is independent from the axion coupling. As $\beta_{A} \approx 10^{-3}$ in the nonrelativistic regime, the velocities cancel out in the convolution between $\sigma_{\mathrm{Ae}}$ and the flux. Thus, the expected electron recoil spectrum is independent from the particle speed. As the kinetic energy of the ALPs is negligible with respect to its rest mass, a monoenergetic peak at the axion mass is expected in the spectrum.

\section{XENON100}

The XENON100 experiment's primary aim is to detect dark matter in form of weakly interactive massive particle (WIMP) through their elastic nuclear scattering off nuclei in the LXe target. The detector is a cylindrical $(30-\mathrm{cm}$ height $\times 30-\mathrm{cm}$ diameter) dual-phase time-projection chamber (TPC) with $62 \mathrm{~kg}$ of LXe, employed both as target and detection medium. It operates at the Laboratori Nazionali del Gran Sasso (LNGS). The detector is equipped with 242 radio pure photomultiplier tubes (PMTs) placed on top (in the xenon gas) and on the bottom of the TPC (immersed in the LXe below the cathode). A particle interaction in the LXe target creates both excited and ionized atoms. De-excitation leads to a prompt scintillation signal $(S 1)$. Due to the presence of an electric drift field of $530 \mathrm{~V} / \mathrm{cm}$, a large fraction of the ionization electrons is drifted away from the interaction site and extracted from the liquid into the gas phase by a strong extraction field of $\sim 12 \mathrm{kV} / \mathrm{cm}$, generating a light signal $(S 2)$ by proportional scintillation in the gas. Threedimensional event vertex reconstruction is achieved using the time difference between the $S 1$ and the $S 2$ signals along with the $S 2$ hit pattern on the top PMTs, which is employed to estimate the $(x, y)$ coordinate. The $S 1$ signal is used to estimate the energy deposited in the detector, as explained below [Eq. (2)]. A detailed description of the instrument is given in [19].

The ratio $S 2 / S 1$ is different whether the energy deposit in the LXe is due to electronic recoil (ER) or to nuclear recoil (NR). Therefore, this $S 2 / S 1$ ratio is used to discriminate the two topologies of events. In the case of ERs, such as from interaction with $\gamma, \beta$ backgrounds and axion signals, the energy from the incoming particle is transferred to the electrons of the Xe atom. Conversely, neutrons or WIMPs scatter off the Xe nuclei. The total background in the inner 34-kg superellipsoidal fiducial volume of the LXe target corresponds to $5.3 \times 10^{-3}$ events $/(\mathrm{keV} \times \mathrm{kg} \times$ day $)$ [20], making XENON100 extremely sensitive to rare event searches in general. The ultralow background has been achieved by means of several techniques: the careful selection of materials [21]; the detector design, with radioactive parts far away from the target; the powerful passive shield, as well as an active LXe veto; and the selfshielding power of LXe, exploited by selecting only the inner part of the TPC for the analysis. The background is dominated by Compton events which scatter only once in the low-energetic region of interest, resulting in an almost flat spectrum [22]. Under an average depth of 3600-m 
water equivalent, the cosmic muon flux is suppressed by 6 orders of magnitude with respect to sea level.

\section{ANALYSIS}

\section{A. Data sample and analysis}

In this work, we analyze the same data set used for the spin-independent [20] and spin-dependent [23] WIMPsearches, with an exposure of 224.6 live days and 34-kg fiducial mass. Two main classes of analysis cuts have been applied. The first one consists of basic data quality selection to remove either unidentified energy deposition peaks or excessive electronic noise level. Since only singlescatter events are expected from axion interactions, the second class of cuts identifies such events by using the number of $S 1$ and $S 2$ peaks. Conditions on the size of the $S 2$ and the requirement that at least two PMTs must observe an $S 1$ signal ensure that only data above the threshold and well above the noise level are considered. Finally, consistency criteria are applied. These are identical to the one used in the above mentioned WIMP searches, with the exception of a cut on the $S 2$ width. The original definition of this consistency cut, comparing the width of the proportional $S 2$ peak to its time delay with respect to the $S 1$, had been found to not be useful for this analysis targeted at ERs and was hence not used. Detailed information on the procedure is available in [24].

Figure 1 (top) shows the distribution in the $\log _{10}\left(S 2_{b} / S 1\right)$ vs $S 1$ for calibration data (grey dots) and
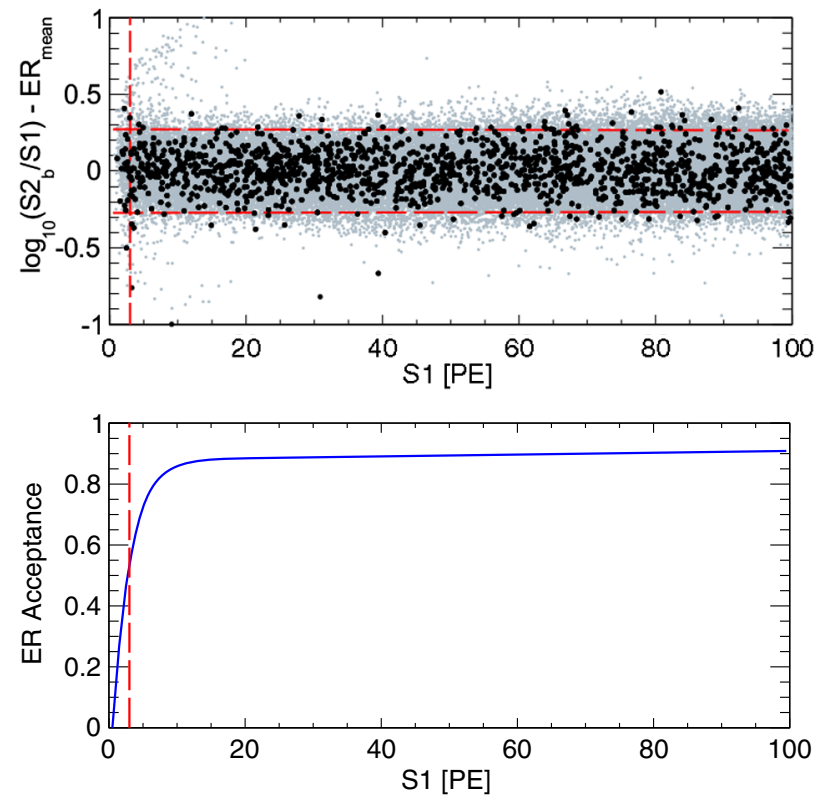

FIG. 1 (color online). (Top) Event distribution in the flattened $\log _{10}\left(S 2_{b} / S 1\right)$ vs $S 1$ space for science data (black points) and calibration (grey points). Straight dashed lines show the selection cut on the flattened $\log _{10}\left(S 2_{b} / S 1\right)$ (horizontal red lines) and the three PE threshold cut (red vertical line). (Bottom) Global acceptance for ER events, evaluated on calibration data. the science data passing all of the selection cuts (black dots). Only the $S 2$ signal detected by the bottom PMTs, $S 2_{b}$, is used since it requires smaller corrections [19]. The calibration data are obtained by exposing the detector to ${ }^{60} \mathrm{Co}$ and ${ }^{232} \mathrm{Th}$ sources. These have been chosen, as their high-energy gamma rays can penetrate the LXe into the fiducial volume, leaving a low-energetic Compton scatter spectrum covering the energy region of interest. The mean of the $\log _{10}\left(S 2_{b} / S 1\right)$ band from the calibration is subtracted in order to remove the energy dependence of this parameter. The lower energy threshold was set to three photoelectrons (PEs) in $S 1$ ( $2 \mathrm{keV}$ for ER energy deposit) in order to limit the presence of random coincidences from dark counts in the PMTs. In addition, a lower threshold of $150 \mathrm{PE}$ in $S 2$ has been imposed to be unaffected by the trigger threshold [24].

In order to reject ER events with an anomalously high or low $S 2 / S 1$ ratio, signal candidates are required to be inside the $2 \sigma$ band around the $\log _{10}\left(S 2_{b} / S 1\right)$ median [24]. This is shown by the horizontal red dashed lines in Fig. 1 (top). The combined acceptance of all selection cuts for ER events is evaluated on calibration data and is shown in Fig. 1 (bottom). Upper thresholds of 30 and 100 PE were employed for the axions from the Sun and the nonrelativistic ALPs searches, respectively.

The energy deposited by each interaction is obtained using the observed $S 1$ signal. The keV-PE conversion is performed using the noble element simulation technique (NEST) model (v 0.98) [25]. This takes into account the scintillation efficiency $R(E)$ relative to the $32.1-\mathrm{keV}$ transition of ${ }^{83 m} \mathrm{Kr}$ at zero electric field (as chosen by [26] and [27]) and the quenching factor $Q(E)$ for a nonzero electric field (measured by [27] for values close to the field applied in XENON100). The model agrees with the direct measurements at zero field [26,27], as well as the measurements with a nonzero field $[27,28]$. The uncertainty on $R(E) \times Q(E)$ is taken from NEST and is assumed to be Gaussian. This reflects the intrinsic uncertainty of the model (4\%), as well as the spread in the measured data points, particularly relevant at low energies. The conversion from the energy deposition $E$ to the observed signal $n^{\exp }$ in $\mathrm{PE}$ is therefore given by

$$
n^{\exp }(E)=R(E) \times Q(E) \times f \times E \equiv L_{Y}(E) \times E,
$$

where the factor $f=3.76 \mathrm{PE} / \mathrm{keV}$ is the derived XENON100 light yield at $32.1 \mathrm{keV}$ and zero field $[19,28]$. The function $n^{\exp }(E)$ is shown in Fig. 2, together with the $\pm 1 \sigma$ uncertainty.

\section{B. Statistical method}

A profile likelihood analysis, as described in [29] and analogous to [30], is used to constrain the coupling constant $g_{\mathrm{Ae}}$. The full likelihood function is given by

$$
\mathcal{L}=\mathcal{L}_{1}\left(g_{\mathrm{Ae}}, N_{b}, n^{\exp }\right) \times \mathcal{L}_{2}\left(n^{\exp }\right) .
$$


E. APRILE et al.

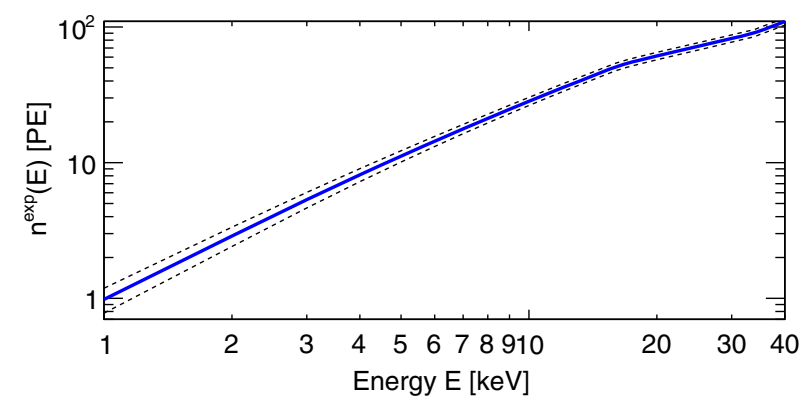

FIG. 2 (color online). Conversion function between energy recoil in $\mathrm{keV}$ and $S 1$ in PE. The $n^{\exp }$ central value and the $\pm 1 \sigma$ uncertainty are indicated with solid blue and black dashed line, respectively.

The parameter of interest is $g_{\mathrm{Ae}}$, whereas $N_{b}$ and $n^{\exp }$ are considered as nuisance parameters. The first term,

$$
\mathcal{L}_{1}=\operatorname{Poiss}\left(N \mid N_{s}+N_{b}\right) \prod_{i=1}^{N} \frac{N_{s} f_{s}\left(S 1_{i}\right)+N_{b} f_{b}\left(S 1_{i}\right)}{N_{s}+N_{b}},
$$

describes the measurement of the detector. The second term,

$$
\mathcal{L}_{2}\left(n^{\exp }(t)\right)=\mathrm{e}^{-t^{2} / 2},
$$

is used to constrain the energy scale.

The energy scale term, $\mathcal{L}_{2}$, has been parametrized with a single parameter $t$. The likelihood function is defined to be normally distributed with zero mean and unit variance, corresponding to where $t= \pm 1$ corresponds to a $\pm 1 \sigma$ deviation in $n^{\text {exp }}$, as shown in Fig. 2 ; i.e., $t=\left(n^{\exp }-n_{\text {mean }}^{\exp }\right) / \sigma$.

In Eq. (4), $N_{s}$ and $N_{b}$ are the expected number of signal and background events in the search region, and $N_{s}$ depends on $g_{\mathrm{Ae}}$ and $n^{\exp } . N$ is the total number of observed events, and the $S 1_{i}$ corresponds to the $S 1$ of the $i$ th event. The functions $f_{s}$ and $f_{b}$ are the normalized signal and background probability distribution functions.

The event rate with a given number of detected photons, $n$, is obtained by applying Poisson smearing to the predicted energy spectrum $d R / d E$,

$$
\frac{d R}{d n}=\int_{0}^{\infty} \frac{d R}{d E} \times \operatorname{Poiss}\left(n \mid n^{\exp }(E)\right) d E
$$

where $n^{\exp }$ is obtained from Eq. (2).

The rate as a function of the measured number of PEs, $S 1$, is given by

$$
\frac{d R}{d S 1}=\sum_{n=1}^{\infty} \operatorname{Gauss}\left(S 1 \mid n, \sqrt{n} \sigma_{\mathrm{PMT}}\right) \times \frac{d R}{d n} \times \epsilon(S 1),
$$

PHYSICAL REVIEW D 90, 062009 (2014)

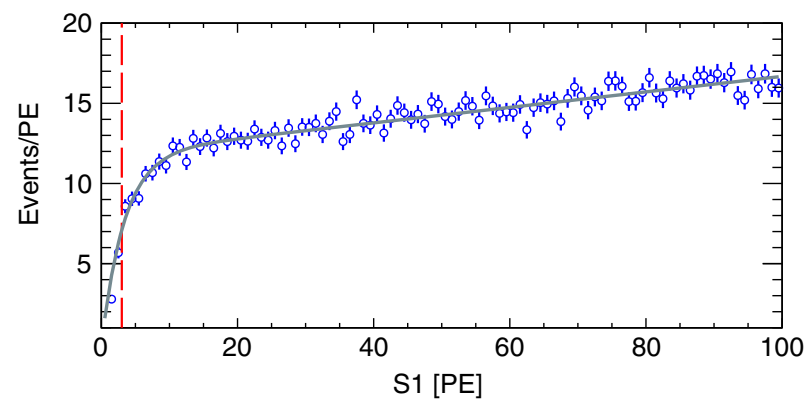

FIG. 3 (color online). Background model $N_{b} \times f_{b}$ (grey line), scaled to the correct exposure, as explained in the text. The analytic function $f_{b}$ is based on the ${ }^{60} \mathrm{Co}$ and ${ }^{232} \mathrm{Th}$ calibration data (empty blue dots) and is used in Eq. (4). The three-PE threshold is indicated by the vertical red dashed line.

where $\sigma_{\mathrm{PMT}}=0.5 \mathrm{PE}$ is the PMT resolution [24], and $\epsilon(S 1)$ is the acceptance of all criteria applied to the data [see Fig. 1 (bottom)]. It has a rather flat behavior above 10 PE. Below that, the acceptance decreases mainly due to data quality criteria.

The majority of the background events arises from gamma scattering off the atomic electrons of the LXe target, as well from intrinsic beta background ${ }^{222} \mathrm{Rn}$ and ${ }^{85} \mathrm{Kr}$ ) [22]. To model these events, we use the ${ }^{60} \mathrm{Co}$ and ${ }^{222} \mathrm{Th}$ calibration data. The total spectrum is then analytically parametrized by means of a modified Fermi function, $f_{b}(S 1)$, shown in Fig. 3 (grey line) along with the calibration data (empty blue dots). The spectrum is scaled to the science data exposure by normalizing it to the number of events seen outside the signal region to avoid biases. For solar axions, it is done between 30 and $100 \mathrm{PE}$, and for galactic ALPs below $m_{A}[\mathrm{pe}]-2 \sigma$ and above $m_{A}[\mathrm{pe}]+2 \sigma$, where $m_{A}[\mathrm{pe}]$ is the ALP mass in units of $\mathrm{PE}$ and $\sigma$ is the width of the expected signal peak (see Fig. 6). Then the scaled background spectrum is integrated in the signal region to give the expected number of background events, $N_{b}$. The background model scaled to the correct exposure, $N_{b} \times f_{b}$, is shown in Fig. 3, along with the scaled calibration spectrum.

As downward statistical fluctuations of the background might lead to reject couplings to which the experiment is not sensitive, we used the $\mathrm{CL}_{\mathrm{s}}$ method to protect the result from this effect, as described in [30].

\section{RESULTS}

\section{A. Solar axions}

The spectrum of the remaining 393 events, between 3 and $30 \mathrm{PE}$ and after all the selection cuts, is shown in Fig. 4 as a function of $S 1$. The solid grey line shows the background model, $N_{b} \times f_{b}$. The expected $S 1$ spectrum for solar axions, lighter than $1 \mathrm{keV} / \mathrm{c}^{2}$, is shown as a blue dashed line for $g_{\mathrm{Ae}}=2 \times 10^{-11}$, i.e., the best limit so far, reported by the EDELWEISS-II Collaboration [31]. 


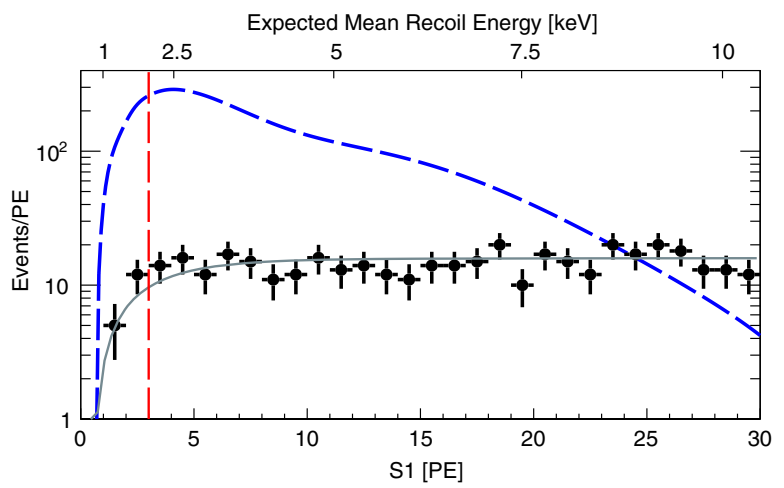

FIG. 4 (color online). Event distribution of the data (black dots) and background model (grey) of the solar axion search. The expected signal for solar axions with $m_{A}<1 \mathrm{keV} / \mathrm{c}^{2}$ is shown by the dashed blue line, assuming $g_{\mathrm{Ae}}=2 \times 10^{-11}$, the current best limit, from EDELWEISS-II [31]. The vertical dashed red line indicates the low $S 1$ threshold, set at three PE. The top axis indicates the expected mean energy for ERs as derived from the observed S1 signal.

The data are compatible with the background model, and no excess is observed for the background only hypothesis.

Figure 5 shows the new XENON100 exclusion limit on $g_{\mathrm{Ae}}$ at $90 \%$ C.L. The sensitivity is shown by the green/ yellow band $(1 \sigma / 2 \sigma)$. As we used the most recent and accurate calculation for solar axion flux from [10], which is valid only for light axions, we restrict the search to $m_{A}<1 \mathrm{keV} / \mathrm{c}^{2}$. For comparison, we also present other recent experimental constraints [31-33]. Astrophysical

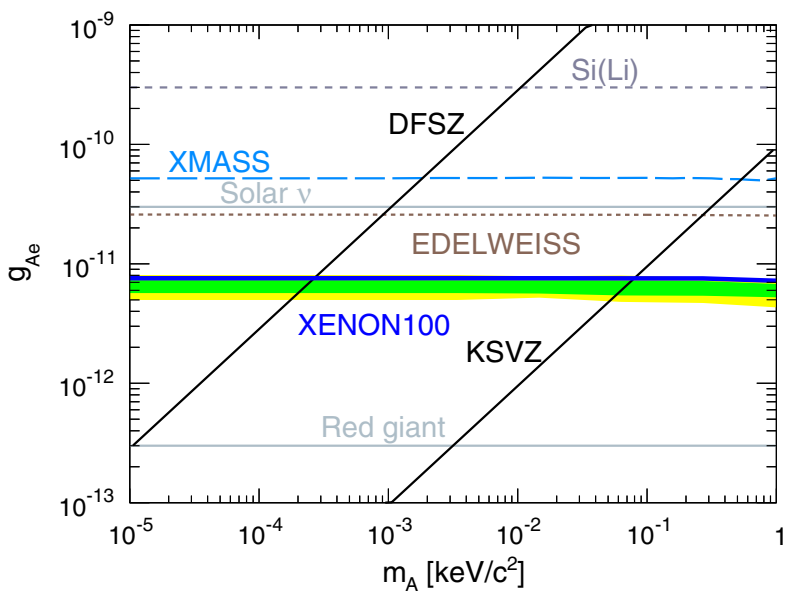

FIG. 5 (color online). The XENON100 limits (90\% C.L.) on solar axions are indicated by the blue line. The expected sensitivity, based on the background hypothesis, is shown by the green/yellow bands $(1 \sigma / 2 \sigma)$ around the XENON100 limits. Results by EDELWEISS-II [31] and XMASS [32] are shown, together with the ones from a $\mathrm{Si}(\mathrm{Li})$ detector by Derbin et al. [33]. Indirect astrophysical bounds from solar neutrinos [34] and red giants [35] are represented by light grey horizontal lines. The benchmark DFSZ and KSVZ models are represented by dark grey lines [4-7]. bounds [34-36] and theoretical benchmark models [4-7] are also shown. For solar axions with masses below $1 \mathrm{keV} / \mathrm{c}^{2}, \mathrm{XENON100}$ is able to set the strongest constraint on the coupling to electrons, excluding values of $g_{\mathrm{Ae}}$ larger than $7.7 \times 10^{-12}$ (90\% C.L.).

For a specific axion model, the limit on the dimensionless coupling $g_{\mathrm{Ae}}$ can be translated to a limit on the axion mass. Within the DFSZ and KSVZ models [4-7], XENON100 excludes axion masses above $0.3 \mathrm{eV} / \mathrm{c}^{2}$ and $80 \mathrm{eV} / \mathrm{c}^{2}$, respectively. For comparison, the CAST experiment, testing the coupling to photons, $g_{A \gamma}$, has excluded axions within the KSVZ model in the mass range between 0.64 and $1.17 \mathrm{eV} / \mathrm{c}^{2}[37,38]$.

\section{B. Galactic ALPs}

Figure 6 shows the XENON100 data after the selection cuts in the larger energy region of interest used for the search for nonrelativistic galactic ALPs (1422 surviving events), along with their statistical errors. Also shown is the expected signal for different ALP masses, assuming a coupling of $g_{\mathrm{Ae}}=4 \times 10^{-12}$ and that ALPs constitute all of the galactic dark matter. The width of the monoenergetic signal is given by the energy resolution of the detector at the relevant $S 1$ signal size [19]. As for the solar axion search, the data are compatible with the background hypothesis, and no excess is observed for the background-only hypothesis for the various ALP masses.

The XENON100 90\% C.L. exclusion limit for galactic ALPs is shown in Fig. 7, together with other experimental constraints $[31,39,40]$. Astrophysical bounds [34-36] and the KSVZ benchmark model [6,7] are also presented. The expected sensitivity is shown by the green/yellow bands $(1 \sigma / 2 \sigma)$. The steps in the sensitivity around 5 and $35 \mathrm{keV} / \mathrm{c}^{2}$ reflect the photoelectric cross section due to

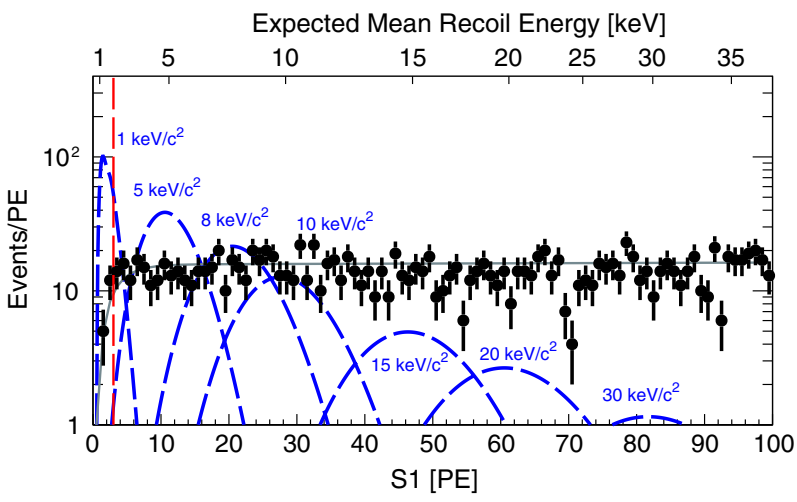

FIG. 6 (color online). Event distribution in the galactic ALPs search region between 3 and 100 PE (black dots with error bars). The grey line shows the background model used for the profile likelihood function. The vertical dashed red line indicates the $S 1$ threshold. The expected signal in XENON100 for various ALP masses, assuming $g_{\mathrm{Ae}}=4 \times 10^{-12}$, is shown as blue dashed peaks. The top axis indicates the expected mean energy for ERs as derived from the observed $S 1$ signal. 


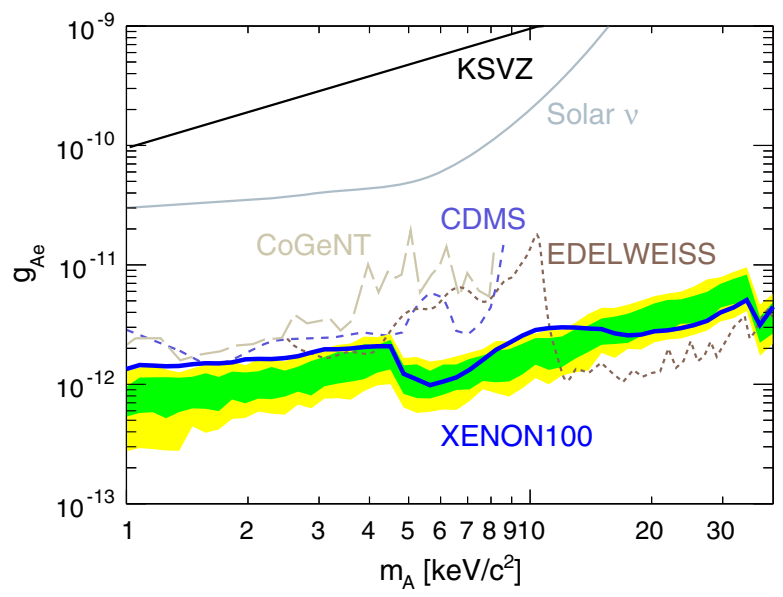

FIG. 7 (color online). The XENON100 limits (90\% C.L.) on ALP coupling to electrons as a function of the mass, under the assumption that ALPs constitute all of the dark matter in our galaxy (blue line). The expected sensitivity is shown by the green/ yellow bands $1 \sigma / 2 \sigma$. The other curves are constraints set by CoGeNT [39] (light brown dashed line), CDMS [40] (blue dashed line, more dotted), and EDELWEISS-II [31] (ochre dashed line, extending up to $40 \mathrm{keV} / \mathrm{c}^{2}$ ). Indirect astrophysical bound from solar neutrinos [34] is represented as a continuous light grey line. The benchmark KSVZ model is represented by a dark grey line $[6,7]$.

the atomic energy levels. Below $5 \mathrm{keV} / \mathrm{c}^{2}$ the obtained $90 \%$ C.L. is higher than expected, deviating by as much as $2 \sigma$ from the mean predicted sensitivity. This is due to a slight excess of events between 3 and 5 PE. A similar effect is responsible for the limit oscillating around the predicted sensitivity above $5 \mathrm{keV} / \mathrm{c}^{2}$. The ALP limit is very sensitive to fluctuations in individual bins because of the expected monoenergetic signal. In the $5-10 \mathrm{keV} / \mathrm{c}^{2}$ mass range, XENON100 sets the best upper limit, excluding an axionelectron coupling $g_{\mathrm{Ae}}>1 \times 10^{-12}$ at the $90 \%$ C.L., assuming that ALPs constitute all of the galactic dark matter.

The impact of systematic uncertainties has been evaluated for both analyses presented here. In particular, we have considered the parametrization of the cross section of the axioelectric effect, the data selection based on a band in the $\log _{10}\left(S 2_{b} / S 1\right)$ vs $S 1$ space, the choice of the fiducial volume, as well as the conversion of the $S 1$ signal into an ER energy and the energy resolution.

Previous works (e.g., [15,32]) have used a different parametrization of the axion velocity term in $\sigma_{A}$, while we chose to employ $\left(1-\beta_{A}^{2 / 3} / 3\right)$ [Eq. (1)], as suggested by [31]. However, we also tested the other assumptions and found the impact on the final limit to be negligible.

Varying the width of the band chosen to select the data entering the analysis [shown in Fig. 1 (top) as horizontal dashed red lines] from $\pm 1 \sigma$ up to $\pm 4 \sigma$ changes the final result on $g_{\mathrm{Ae}}$ by $5 \%$, i.e., well within the $\pm 2 \sigma$ of the sensitivity band.

Similarly, a variation of the fiducial volume has a negligible impact on the sensitivity: the inner ellipsoid was changed in size to accomodate between 28 and $40 \mathrm{~kg}$, but maintaining the same 224.6 days of live time. The reduced background for smaller fiducial masses is compensated by the smaller total exposure, resulting in a variation of the limit well below $10 \%$.

The uncertainty on the energy scale used for the conversion from the observed $S 1$ signal in PE into $\mathrm{keV}$ [Fig. 2 and Eq. (2)] is taken into account in the profile likelihood function and is profiled out via the nuisance parameter $t$ [Eq. (5)]. The detector's energy resolution is considered by smearing the predicted energy spectrum $d R / d E$ by Poisson and Gaussian processes, as described in Eq. (7). We note that the final results on $g_{\mathrm{Ae}}$ are also robust against further changes in the energy scale: even if $L_{Y}(E)$, as defined in Eq. (2), is varied by $25 \%$, the limits change by less than $5 \%$ and about $10 \%$ for the solar and for the galactic axion searches, respectively.

\section{ACKNOWLEDGMENTS}

We gratefully acknowledge support from NSF, DOE, SNF, Volkswagen Foundation, FCT, Region des Pays de la Loire, STCSM, NSFC, DFG, MPG, Stichting voor Fundamenteel Onderzoek der Materie (FOM), the Weizmann Institute of Science, the EMG research center, and INFN. We are grateful to LNGS for hosting and supporting XENON100.
[1] R. D. Peccei and H. R. Quinn, Phys. Rev. Lett. 1440, 38 (1977).

[2] S. Weinberg, Phys. Rev. Lett. 40, 223 (1978).

[3] F. Wilczeck, Phys. Rev. Lett. 40, 279 (1978).

[4] M. Dine, W. Fischler, and M. Srednicki, Phys. Lett. 104B, 199 (1981).

[5] A. R. Zhitnitsky, Sov. J. Nucl. Phys. 31, 260 (1980).

[6] J. E. Kim, Phys. Rev. Lett. 43, 103 (1979).
[7] M. A. Shifman, A. I. Vainshtein, and V. I. Zakharov, Nucl. Phys. B166, 493 (1980).

[8] L. Abbott and P. Sikivie, Phys. Lett. 120B, 133 (1983).

[9] P. Sikivie, Phys. Rev. Lett. 51, 1415 (1983).

[10] J. Redondo, J. Cosmol. Astropart. Phys. 12 (2013) 008.

[11] S. Dimopoulos, G. D. Starkman, and B. W. Lynn, Phys. Rev. B 168, 145 (1986). 
[12] F. Avignone III, R. Brodzinski, S. Dimopoulos, G. Starkman, A. Drukier, D. Spergel, G. Gelmini, and B. Lynn, Phys. Rev. D 35, 2752 (1987).

[13] M. Pospelov, A. Ritz, and M. Voloshin, Phys. Rev. D 78, 115012 (2008).

[14] A. Derevianko, V. A. Dzuba, V. V. Flambaum, and M. Pospelov Phys. Rev. D 82, 065006 (2010).

[15] K. Arisaka, P. Beltrame, C. Ghag, J. Kaidi, K. Lung, A. Lyashenko, R. D. Peccei, P. Smith, and K. Ye, Astropart. Phys. 44, 59 (2013).

[16] F. Alessandria et al. (CUORE Collaboration), J. Cosmol. Astropart. Phys. 05 (2013) 007.

[17] NIST, http://physics.nist.gov/PhysRefData/Xcom/html/ xcom1.html.

[18] A. M. Green, Mod. Phys. Lett. A 27, 1230004 (2012).

[19] E. Aprile et al. (XENON100 Collaboration), Astropart. Phys. 35, 573 (2012).

[20] E. Aprile et al. (XENON100 Collaboration), Phys. Rev. Lett. 109, 181301 (2012).

[21] E. Aprile et al. (XENON100 Collaboration), Astropart. Phys. 35, 43 (2011).

[22] E. Aprile et al. (XENON100 Collaboration), Phys. Rev. D 83, 082001 (2011).

[23] E. Aprile et al. (XENON100 Collaboration), Phys. Rev. Lett. 111, 021301 (2013).

[24] E. Aprile et al. (XENON100 Collaboration), Astropart. Phys. 54, 11 (2014).

[25] M. Szydagis, N. Barry, K. Kazkaz, J. Mock, D. Stolp, M. Sweany, M. Tripathi, S. Uvarov, N. Walsh, and M. Woods, JINST 6, P10002 (2011).
[26] E. Aprile et al. Phys. Rev. D 86, 112004 (2012).

[27] L. Baudis, H. Dujmovic, C. Geis, A. James, A. Kish, A. Manalaysay, T. Marrodán Undagoitia, and M. Schumann Phys. Rev. D 87, 115015 (2013).

[28] A. Manalaysay et al., Rev. Sci. Instrum. 81, 073303 (2010).

[29] G. Cowan, K. Cranmer, E. Gross, and O. Vitells, Eur. Phys. J. C 71, 1554 (2011).

[30] E. Aprile et al. (XENON100 Collaboration), Phys. Rev. D 84, 052003 (2011).

[31] E. Armengaud et al. (EDELWEISS-II Collaboration), J. Cosmol. Astropart. Phys. 11 (2013) 067.

[32] K. Abe et al. (XMASS Collaboration), Phys. Lett. B 724, 46 (2013).

[33] A. V. Derbin, I. S. Drachnev, A. S. Kayunov, and V. N. Muratova, JETP Lett. 95, 339 (2012).

[34] P. Gondolo and G. G. Raffelt, Phys. Rev. D 79, 107301 (2009).

[35] N. Viaux, M. Catelan, P. B. Stetson, G. G. Raffelt, J. Redondo, A. A. R. Valcarce, and A. Weiss, Phys. Rev. Lett. 111, 231301 (2013).

[36] G. G. Raffelt, Lect. Notes Phys. 741, 51 (2008).

[37] M. Arik et al. (CAST Collaboration), Phys. Rev. Lett. 112, 091302 (2014).

[38] K. Barth et al. (CAST Collaboration), J. Cosmol. Astropart. Phys. 05 (2013) 010.

[39] C. E. Aalseth et al. (CoGeNT Collaboration), Phys. Rev. Lett. 101, 251301 (2008).

[40] Z. Ahmed et al. (CDMS Collaboration), Phys. Rev. Lett. 103, 141802 (2009). 